\title{
Sharp bounds for the arithmetic-geometric mean
}

\section{Zhen-Hang Yang, Ying-Qing Song and Yu-Ming Chu*}

\section{"Correspondence:}

chuyuming2005@126.com

School of Mathematics and

Computation Sciences, Hunan City

University, Yiyang, 413000, China

\section{Abstract}

In this article, we establish some new inequality chains for the ratio of certain bivariate means, and we present several sharp bounds for the arithmetic-geometric mean.

MSC: Primary 26E60; 26D07; secondary 33E05

Keywords: arithmetic-geometric mean; Stolarsky mean; Gini mean; complete elliptic integral of the first kind

\section{Introduction}

Let $\mathbb{R}_{+}$be the set of positive real numbers. Then a two-variable continuous function $M$ : $\mathbb{R}_{+}^{2} \rightarrow \mathbb{R}_{+}$is said to be a mean on $\mathbb{R}_{+}$if the double inequality

$$
\min (a, b) \leq M(a, b) \leq \max (a, b)
$$

holds for all $a, b \in \mathbb{R}_{+}$.

The classical arithmetic-geometric mean $\operatorname{AGM}(a, b)$ of two positive real numbers $a$ and $b$ is defined as the common limit of sequences $\left\{a_{n}\right\}$ and $\left\{b_{n}\right\}$, which are given by

$$
\operatorname{AGM}(a, b)=\lim _{n \rightarrow \infty} a_{n}=\lim _{n \rightarrow \infty} b_{n}
$$

where $a_{0}=a, b_{0}=b$, and for $n \in \mathbb{N}$,

$$
a_{n+1}=\frac{a_{n}+b_{n}}{2}, \quad b_{n+1}=\sqrt{a_{n} b_{n}}
$$

The well-known Gauss identity shows that

$$
\operatorname{AGM}\left(1, \sqrt{1-r^{2}}\right)=\frac{\pi}{2 \mathrm{~K}(r)}
$$

for $r \in(0,1)$, where $\mathrm{K}(r)=\int_{0}^{\pi / 2}\left(1-r^{2} \sin ^{2} t\right)^{-1 / 2} d t[1]$ is the complete elliptic integral of the first kind.

Let $a, b>0$ with $a \neq b$. Then the well-known Stolarsky mean [2] $S_{p, q}(a, b)$ can be expressed as 


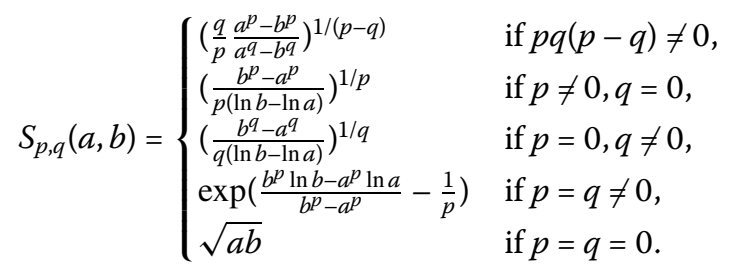

Many bivariate means are the special case of the Stolarsky mean, for example, $S_{2,1}(a, b)=$ $(a+b) / 2=A(a, b)$ is the arithmetic mean, $S_{0,0}(a, b)=\sqrt{a b}=G(a, b)$ is the geometric mean, $S_{3 / 2,1 / 2}(a, b)=(a+\sqrt{a b}+b) / 3=H e(a, b)$ is the Heronian mean, $S_{1,0}(a, b)=(b-a) /(\ln b-$ $\ln a)=L(a, b)$ is the logarithmic mean, $S_{1,1}(a, b)=1 / e\left(b^{b} / a^{a}\right)^{1 /(b-a)}=I(a, b)$ is the identric (exponential) mean, $S_{2 p, p}(a, b)=A^{1 / p}\left(a^{p}, b^{p}\right)=A_{p}(a, b)$ the $p$-order arithmetic (power, Hölder) mean, $S_{3 p / 2, p / 2}(a, b)=H e^{1 / p}\left(a^{p}, b^{p}\right)=H e_{p}(a, b)$ is the $p$-order Heronian mean, $S_{p, 0}(a, b)=L^{1 / p}\left(a^{p}, b^{p}\right)=L_{p}(a, b)$ is the $p$-order logarithmic mean, $S_{p, p}(a, b)=I^{1 / p}\left(a^{p}, b^{p}\right)=$ $I_{p}(a, b)$ is the $p$-order identric (exponential) mean and $S_{p+1, p}(a, b)=\left[p\left(a^{p+1}-b^{p+1}\right)\right] /[(p+$ 1) $\left.\left(a^{p}-b^{p}\right)\right]=\mathcal{J}_{p}(a, b)$ is the one-parameter mean.

Another important family of means is the Gini means [3] defined by

$$
G_{p, q}(a, b)= \begin{cases}\left(\frac{a^{p}+b^{p}}{a^{q}+b^{q}}\right)^{1 /(p-q)} & \text { if } p \neq q, \\ \exp \left(\frac{a^{p} \ln a b^{p} \ln b}{a^{p}+b^{p}}\right) & \text { if } p=q,\end{cases}
$$

it also contains many special means, for instance, $G_{1,0}(a, b)=A(a, b)$ is the arithmetic mean, $G_{1,1}(a, b)=a^{a /(a+b)} b^{b /(a+b)}=I\left(a^{2}, b^{2}\right) / I(a, b)=Z(a, b)$ is the power-exponential mean, $G_{p, 0}(a, b)=A^{1 / p}\left(a^{p}, b^{p}\right)=A_{p}(a, b)$ is the $p$-order arithmetic (power, Hölder) mean, $G_{p, p}(a, b)=Z^{1 / p}\left(a^{p}, b^{p}\right)=Z_{p}(a, b)$ is the $p$-order power-exponential mean and $G_{p+1, p}(a, b)=$ $\left(a^{p+1}+b^{p+1}\right) /\left(a^{p}+b^{p}\right)=\mathcal{L}_{p}(a, b)$ is the Lehmer mean.

Recently, the inequalities for the bivariate means have been the subject of intensive research. In particular, the bounds for the arithmetic-geometric mean $A G M$ have attracted the attention of many mathematicians. It is well known that the double inequality

$$
L(a, b)<A G M(a, b)<L^{2 / 3}\left(a^{3 / 2}, b^{3 / 2}\right)
$$

holds for all $a, b>0$ with $a \neq b$. The first inequality of (1.4) was first proposed by Carlson and Vuorinen [4], it was proved in the literature [5-8] by different methods. Vamanamurthy and Vuorinen [9] (also see [5, 6]) proved that $A G M(a, b)<(\pi / 2) L(a, b)$ for all $a, b>0$ with $a \neq b$. The second inequality of (1.4) is due to Borwein and Borwein [10], and Yang [8] presented a simple proof by use of the 'Comparison Lemma' [10, Lemma 2.1].

In [9] Vamanmurthy and Vuorinen presented the upper bounds for the arithmeticgeometric mean $A G M$ in terms of the arithmetic mean $A$, geometric mean $G$ and logarithmic mean $L$ as follows:

$$
\begin{aligned}
& A G M(a, b)<L_{2}(a, b)=(A(a, b) L(a, b))^{1 / 2}, \\
& A G M(a, b)<I(a, b)<A(a, b), \\
& A G M(a, b)<A_{1 / 2}(a, b)
\end{aligned}
$$

for all $a, b>0$ with $a \neq b$. 
In 1995, Sándor [5] proved that the double inequality

$$
\frac{1}{\frac{12 /(5 \pi)}{L(a, b)}+\frac{1-12 /(5 \pi)}{A(a, b)}}<A G M(a, b)<\frac{1}{\frac{2 / \pi}{L(a, b)}+\frac{1-2 / \pi}{A(a, b)}},
$$

holds for all $a, b>0$ with $a \neq b$, and it was improved by Alzer and Qiu [11, Theorem 19] as

$$
\frac{1}{\frac{\beta_{2}}{L(a, b)}+\frac{1-\beta_{2}}{A(a, b)}}<A G M(a, b)<\frac{1}{\frac{\alpha_{2}}{L(a, b)}+\frac{1-\alpha_{2}}{A(a, b)}},
$$

with the best possible parameters $\beta_{2}=3 / 4$ and $\alpha_{2}=2 / \pi$.

Other inequalities involving $A G M$ can be found in the literature [12-20].

The aim of this paper is to establish the new inequality chains for the ratio of certain bivariate means, and we present the sharp bounds for the arithmetic-geometric mean AGM.

\section{Lemmas}

In order to establish our main results we need several lemmas, which we present in this section.

Lemma 1 ([21, Corollary 1.1]) Let $a, b>0$ with $a \neq b$. Then both $S_{p, 2 m-p}(a, b)$ and $G_{p, 2 m-p}(a, b)$ are strictly increasing (decreasing) with respect to $p \in(-\infty, m)(p \in(m, \infty))$ for fixed $m>0$.

Lemma 2 ([22, Theorem 5], [23, Theorem 3.4]) Let $a, b, c, d>0$ with $b / a>d / c \geq 1$. Then the ratio of Stolarsky means $R(p, 2 m-p ; a, b ; c, d)=S_{p, 2 m-p}(a, b) / S_{p, 2 m-p}(c, d)$ is strictly increasing (decreasing) with respect to $p \in(-\infty, m)(p \in(m, \infty))$ for fixed $m>0$.

Lemma 3 ([24, Theorem 4.1]) Let $a, b, c, d>0$ with $b / a>d / c \geq 1$. Then the ratio of Stolarsky means $R(p, q ; a, b ; c, d)=S_{p, q}(a, b) / S_{p, q}(c, d)$ is strictly log-concave (log-convex) with respect to $p \in((|q|-q) / 2, \infty)(p \in(-\infty,-(|q|+q) / 2))$ for fixed $q \in \mathbb{R}$.

From Lemma 3, we have Corollary 1.

Corollary 1 Let $\lambda>0, \alpha \in(0,1)$ and $a, b, c, d>0$ with $b / a>d / c \geq 1$. Then the function

$$
p \mapsto R^{\alpha}(p, 0 ; a, b ; c, d) R^{1-\alpha}\left(\frac{\lambda-\alpha p}{1-\alpha}, 0 ; a, b ; c, d\right)=: r(p)
$$

is strictly increasing in $(0, \lambda)$ and strictly decreasing in $(\lambda, \lambda / \alpha)$.

Proof Let $p_{1}=(\lambda-\alpha p) /(1-\alpha)$. Then

$$
\begin{aligned}
\frac{r^{\prime}(p)}{r(p)}= & \alpha(\ln R(p, 0 ; a, b ; c, d))^{\prime}+(1-\alpha)\left(\ln R\left(p_{1}, 0 ; a, b ; c, d\right)\right)^{\prime} \\
& \times \frac{-\alpha}{1-\alpha} \\
= & \alpha\left((\ln R(p, 0 ; a, b ; c, d))^{\prime}-\left(\ln R\left(p_{1}, 0 ; a, b ; c, d\right)\right)^{\prime}\right)
\end{aligned}
$$




$$
\begin{aligned}
& =\alpha\left(p-p_{1}\right)(\ln R(\xi, 0 ; a, b ; c, d))^{\prime \prime} \\
& =\frac{\alpha}{1-\alpha}(p-\lambda)(\ln R(\xi, 0 ; a, b ; c, d))^{\prime \prime}
\end{aligned}
$$

where $\xi$ is between $p$ and $p_{1}$.

It follows from Lemma 3 that $R(p, 0 ; a, b ; c, d)$ is strictly log-concave with respect to $p \in$ $(0, \infty)$ and strictly log-convex with respect to $p \in(-\infty, 0)$. Therefore, $r^{\prime}(p)>0$ for $p \in(0, \lambda)$ and $r^{\prime}(p)<0$ for $p \in(\lambda, \lambda / \alpha)$.

Lemma 4 ([25, Corollary 3.1]) Let $a, b, c, d>0$ with $b / a>d / c \geq 1$. Then the function

$$
Q(p)=\frac{\sqrt{S_{p, q}(a, b) S_{2 k-p, q}(a, b)}}{\sqrt{S_{p, q}(c, d) S_{2 k-p, q}(c, d)}}
$$

is strictly decreasing (increasing) in $(k, \infty)$ and strictly increasing (decreasing) in $(-\infty, k)$ for fixed $q \geq(\leq) 0, k \geq(\leq) 0$ with $q^{2}+k^{2} \neq 0$.

Let $(k, q)=(3 / 2,0),(1 / 2,1)$, respectively. Then Lemma 4 leads to the following.

Corollary 2 Let $a, b, c, d>0$ with $b / a>d / c \geq 1$. Then

(i) the function

$$
p \mapsto \frac{\sqrt{S_{p, 0}(a, b) S_{3-p, 0}(a, b)}}{\sqrt{S_{p, 0}(c, d) S_{3-p, 0}(c, d)}}
$$

is strictly decreasing in $(3 / 2, \infty)$ and strictly increasing in $(-\infty, 3 / 2)$;

(ii) the function

$$
p \mapsto \frac{\sqrt{S_{p, 1}(a, b) S_{1-p, 1}(a, b)}}{\sqrt{S_{p, 1}(c, d) S_{1-p, 1}(c, d)}}
$$

is strictly decreasing in $(1 / 2, \infty)$ and increasing in $(-\infty, 1 / 2)$.

Lemma 5 Let $a, b>0$ with $b>a$. Then $b / a>A(a, b) / G(a, b)>1$.

Proof Simple computations lead to

$$
\begin{aligned}
\frac{b}{a}-\frac{A(a, b)}{G(a, b)} & =\frac{b}{a}-\frac{\sqrt{\frac{b}{a}}+\sqrt{\frac{a}{b}}}{2} \\
& =\frac{1}{2}\left(\sqrt{\frac{b}{a}}-1\right)\left(1+2 \sqrt{\frac{b}{a}}+\sqrt{\frac{a}{b}}\right)>0 .
\end{aligned}
$$

Lemma 6 ([26]) Let $x \in(0,1)$. Then

$$
\begin{aligned}
& \operatorname{AGM}(1, x) \sim \frac{\pi / 2}{\ln (1 / x)}, \quad x \rightarrow 0^{+}, \\
& \frac{1}{A G M(1,1-x)}=1+\frac{1}{2} x+\frac{5}{16} x^{2}+\frac{7}{32} x^{3}+\frac{169}{1,024} x^{4}+o\left(x^{4}\right) .
\end{aligned}
$$


Lemma 7 is a consequence of the 'Comparison Lemma' in [10, Lemma 2.1].

Lemma 7 Let $\Phi$ be a bivariate mean such that $\Phi(G(x, y), A(x, y))<(>) \Phi(x, y)$ for all $x, y>0$ with $x \neq y$. Then

$$
\operatorname{AGM}(a, b)<(>) \Phi(a, b)
$$

for all $a, b>0$ with $a \neq b$.

\section{Inequality chains for the ratio of means}

In this section, we give some inequality chains for the ratio of certain bivariate means, which will be used to prove our main results in next section.

Proposition 1 Let $a, b, c, d>0$ with $b / a>d / c \geq 1$. Then we have

$$
\frac{\sqrt{A(a, b) G(a, b)}}{\sqrt{A(c, d) G(c, d)}}<\frac{G_{3 / 4,-1 / 4}(a, b)}{G_{3 / 4,-1 / 4}(c, d)}<\frac{S_{7 / 4,-1 / 4}(a, b)}{S_{7 / 4,-1 / 4}(c, d)} .
$$

Proof The second inequality of (3.1) can be rewritten as

$$
\frac{S_{7 / 4,-1 / 4}(1, b / a)}{G_{3 / 4,-1 / 4}(1, b / a)}>\frac{S_{7 / 4,-1 / 4}(1, d / c)}{G_{3 / 4,-1 / 4}(1, d / c)} .
$$

Therefore, it suffices to prove that the function

$$
f_{1}(x)=\ln \frac{S_{7 / 4,-1 / 4}(1, x)}{G_{3 / 4,-1 / 4}(1, x)}=\frac{1}{2} \ln \frac{x^{7 / 4}-1}{7\left(1-x^{-1 / 4}\right)}-\ln \frac{x^{3 / 4}+1}{x^{-1 / 4}+1}
$$

is strictly increasing in $(1, \infty)$. Replacing $x$ by $x^{4}$ and differentiating $f_{1}$ give

$$
\begin{aligned}
4 x^{3} f_{1}^{\prime}\left(x^{4}\right) & =\frac{7}{2} \frac{x^{6}}{\left(x^{7}-1\right)}-\frac{1}{2(x-1)}-\frac{1}{2 x}-\frac{3 x^{2}}{x^{3}+1}+\frac{1}{x+1} \\
& =\frac{(x+1)\left(x+x^{2}+1\right)(x-1)^{5}}{2 x\left(x^{2}-x+1\right)\left(x^{6}+x^{5}+x^{4}+x^{3}+x^{2}+x+1\right)}>0
\end{aligned}
$$

for $x \in(1, \infty)$.

Similarly, to prove the first inequality of (3.1), it suffices to prove that the function

$$
f_{2}(x)=\ln \frac{G_{3 / 4,-1 / 4}(1, x)}{\sqrt{A(1, x) G(1, x)}}=\ln \frac{x^{3 / 4}+1}{\left(x^{-1 / 4}+1\right) \sqrt{\frac{x+1}{2} \sqrt{x}}}=\ln \frac{\sqrt{x}-\sqrt[4]{x}+1}{\sqrt{\frac{x+1}{2}}}
$$

is strictly increasing on $(1, \infty)$. Replacing $x$ by $x^{4}$ and differentiating $f_{2}$ yield

$$
4 x^{3} f_{2}^{\prime}\left(x^{4}\right)=\frac{(x+1)(x-1)^{3}}{\left(x^{2}-x+1\right)\left(x^{4}+1\right)}>0
$$

for $x \in(1, \infty)$, which completes the proof. 
Proposition 2 Let $a, b>0$ with $a \neq b$. Then we have

$$
\frac{\sqrt{A(a, b) G(a, b)}}{\sqrt{A(G, A) G(G, A)}}<\frac{G_{3 / 4,-1 / 4}(a, b)}{G_{3 / 4,-1 / 4}(G, A)}<\frac{S_{7 / 4,-1 / 4}(a, b)}{S_{7 / 4,-1 / 4}(G, A)}<1,
$$

where $G=G(a, b)$ and $A=A(a, b)$.

Proof By symmetry, without loss of generality, we assume that $a<b$. Then from Lemma 5 and Proposition 1 we clearly see that the first and second inequalities of (3.2) hold. Next we prove the last inequality of (3.2). Let $t=\ln \sqrt{b / a}>0$, then the last inequality of (3.2) can be rewritten as

$$
\left(\frac{\sinh \frac{7 t}{4}}{7 \sinh \frac{t}{4}}\right)^{1 / 2}<\left(\frac{1}{7} \frac{(\cosh t)^{7 / 4}-1}{1-(\cosh t)^{-1 / 4}}\right)^{1 / 2}=\left(\frac{1}{7} \frac{(\cosh t)^{2}-(\cosh t)^{1 / 4}}{(\cosh t)^{1 / 4}-1}\right)^{1 / 2}
$$

It suffices to prove that the function

$$
g(t)=\cosh t-\left(\frac{\sinh \frac{7 t}{4}+\left(\sinh \frac{t}{4}\right)(\cosh t)^{2}}{\sinh \frac{7 t}{4}+\sinh \frac{t}{4}}\right)^{4}<0
$$

for $t>0$.

Simple computations lead to

$$
\begin{aligned}
& \sinh 7 t+(\sinh t)(\cosh 4 t)^{2}=8\left(\sinh t \cosh ^{2} t\right)\left(2 \cosh ^{2} t-1\right)\left(4 \cosh ^{4} t-2 \cosh ^{2} t-1\right), \\
& \sinh 7 t+\sinh t=8\left(\sinh t \cosh ^{2} t\right)\left(4 \cosh ^{2} t-3\right)\left(2 \cosh ^{2} t-1\right), \\
& \cosh 4 t=8 \cosh ^{4} t-8 \cosh ^{2} t+1, \\
& g(4 t)=\cosh 4 t-\left(\frac{\sinh 7 t+(\sinh t)(\cosh 4 t)^{2}}{\sinh 7 t+\sinh t}\right)^{4} \\
& \quad=\left(8 \cosh ^{4} t-8 \cosh ^{2} t+1\right)-\left(\frac{4 \cosh ^{4} t-2 \cosh ^{2} t-1}{4 \cosh ^{2} t-3}\right)^{4}:=g_{1}\left(\cosh ^{2} t\right),
\end{aligned}
$$

where

$$
g_{1}(x)=\left(8 x^{2}-8 x+1\right)-\left(\frac{4 x^{2}-2 x-1}{4 x-3}\right)^{4}, \quad x=\cosh ^{2} t>1 .
$$

$g_{1}(x)$ can be rewritten as

$$
\begin{aligned}
g_{1}(x) & =-16(x-1)^{4} \frac{16 x^{4}+32 x^{3}-88 x^{2}+48 x-5}{(4 x-3)^{4}} \\
& =-16(x-1)^{4} \frac{16 x^{2}(x-1)^{2}+64 x(x-1)^{2}+16 x(x-1)+5\left(x^{2}-1\right)+3 x^{2}}{(4 x-3)^{4}}<0
\end{aligned}
$$

for $x>1$. Therefore, $g(t)<0$ for $t>0$.

Thus we complete the proof. 
Proposition 3 Let $a, b, c, d>0$ with $b / a>d / c \geq 1$ and $p \in(3 / 2,2)$. Then

$$
\begin{aligned}
\frac{A^{1 / 4}(a, b) L^{3 / 4}(a, b)}{A^{1 / 4}(c, d) L^{3 / 4}(c, d)} & <\frac{\sqrt{L_{p}(a, b) L_{3-p}(a, b)}}{\sqrt{L_{p}(c, d) L_{3-p}(c, d)}}<\frac{L_{3 / 2}(a, b)}{L_{3 / 2}(c, d)} \\
& <\frac{\sqrt{L(a, b) A_{2 / 3}(a, b)}}{\sqrt{L(c, d) A_{2 / 3}(c, d)}}<\frac{\sqrt{L(a, b) I(a, b)}}{\sqrt{L(c, d) I(c, d)}}<\frac{S_{5 / 4,1 / 4}(a, b)}{S_{5 / 4,1 / 4}(c, d)} \\
& <\frac{H e_{3 / 4}(a, b)}{H e_{3 / 4}(c, d)}<\frac{A_{1 / 2}(a, b)}{A_{1 / 2}(c, d)}<\frac{I_{3 / 4}(a, b)}{I_{3 / 4}(c, d)} .
\end{aligned}
$$

Proof (i) From part one of Corollary 2 we see that

$$
\frac{\sqrt{S_{2,0}(a, b) S_{1,0}(a, b)}}{\sqrt{S_{2,0}(c, d) S_{1,0}(c, d)}}<\frac{\sqrt{S_{p, 0}(a, b) S_{3-p, 0}(a, b)}}{\sqrt{S_{p, 0}(c, d) S_{3-p, 0}(c, d)}}<\frac{\sqrt{S_{3 / 2,0}(a, b) S_{3 / 2,0}(a, b)}}{\sqrt{S_{3 / 2,0}(c, d) S_{3 / 2,0}(c, d)}}
$$

for $p \in(3 / 2,2)$.

Therefore, the first and second inequalities of Proposition 3 follow from the above inequalities and $\sqrt{S_{1,0}(a, b) S_{2,0}(a, b)}=A^{1 / 4}(a, b) L^{3 / 4}(a, b)$ together with $S_{3 / 2,0}(a, b)=L_{3 / 2}(a, b)$.

(ii) For the third inequality of Proposition 3. From

$$
A_{2 / 3}(a, b)=S_{4 / 3,2 / 3}(a, b)=\frac{S_{4 / 3,0}^{2}(a, b)}{S_{2 / 3,0}(a, b)}
$$

we clearly see that it suffices to prove

$$
\frac{S_{3 / 2,0}(a, b) \sqrt{S_{2 / 3,0}(a, b)}}{S_{3 / 2,0}(c, d) \sqrt{S_{2 / 3,0}(c, d)}}<\frac{S_{4 / 3,0}(a, b) \sqrt{S_{1,0}(a, b)}}{S_{4 / 3,0}(c, d) \sqrt{S_{1,0}(c, d)}} .
$$

Let $(\alpha, \lambda)=(1 / 3,11 / 9)$. Then Corollary 1 leads to the conclusion that the function

$$
p \mapsto R^{1 / 3}(p, 0 ; a, b ; c, d) R^{2 / 3}\left(\frac{11-3 p}{6}, 0 ; a, b ; c, d\right)=: r(p)
$$

is increasing in $(0,11 / 9)$. Therefore, $r(2 / 3)<r(1)$, that is,

$$
\frac{S_{3 / 2,0}(a, b) \sqrt{S_{2 / 3,0}(a, b)}}{S_{3 / 2,0}(c, d) \sqrt{S_{2 / 3,0}(c, d)}}<\frac{S_{4 / 3,0}(a, b) \sqrt{S_{1,0}(a, b)}}{S_{4 / 3,0}(c, d) \sqrt{S_{1,0}(c, d)}} .
$$

(iii) The fourth inequality of Proposition 3 can be written as $A_{2 / 3}(a, b) / A_{2 / 3}(c, d)<$ $I(a, b) / I(c, d)$, that is, $R(4 / 3,2 / 3 ; a, b ; c, d)<R(1,1 ; a, b ; c, d)$. Let $m=1$, then from Lemma 2 we know that $R(p, 2-p ; a, b ; c, d)$ is strictly decreasing with respect to $p \in(1, \infty)$.

(iv) For the sixth, seventh, and eighth inequalities, let $m=3 / 4$, then Lemma 2 leads to the conclusion that $R(p, 3 / 2-p ; a, b ; c, d)$ is strictly decreasing with respect to $p \in(3 / 4, \infty)$. Consequently,

$$
\begin{aligned}
& R\left(\frac{5}{4}, \frac{3}{2}-\frac{5}{4} ; a, b ; c, d\right)<R\left(\frac{9}{8}, \frac{3}{2}-\frac{9}{8} ; a, b ; c, d\right) \\
& <R\left(1, \frac{3}{2}-1 ; a, b ; c, d\right)<R\left(\frac{3}{4}, \frac{3}{2}-\frac{3}{4} ; a, b ; c, d\right),
\end{aligned}
$$

which gives the desired results. 
(v) Finally, we prove the fifth inequality. It can be written as

$$
\frac{\sqrt{L(1, b / a) I(1, b / a)}}{S_{5 / 4,1 / 4}(1, b / a)}<\frac{\sqrt{L(1, d / c) I(1, d / c)}}{S_{5 / 4,1 / 4}(1, d / c)} .
$$

Thus we need only to prove that the function

$$
h(x)=\ln \frac{\sqrt{L(1, x) I(1, x)}}{S_{5 / 4,1 / 4}(1, x)}
$$

is strictly decreasing in $(1, \infty)$. Let $t=\ln \sqrt{x} \in(0, \infty)$. Then

$$
h(x)=\frac{1}{2} \ln \frac{\sinh t}{t}+\frac{1}{2}\left(\frac{t \cosh t}{\sinh t}-1\right)-\ln \frac{\sinh \frac{5 t}{4}}{5 \sinh \frac{t}{4}}:=h_{1}(t)
$$

Differentiating $h_{1}(t)$ yields

$$
h_{1}^{\prime}(t)=-\frac{h_{2}(t)}{4 t \sinh \frac{1}{4} t \sinh \frac{5}{4} t \sinh ^{2} t}
$$

where

$$
\begin{aligned}
h_{2}(t)= & 2 t^{2} \sinh \frac{t}{4} \sinh \frac{5 t}{4}+2 \sinh \frac{t}{4} \sinh \frac{5 t}{4} \sinh ^{2} t-t \cosh \frac{t}{4} \sinh \frac{5 t}{4} \sinh ^{2} t \\
& +5 t \cosh \frac{5 t}{4} \sinh \frac{t}{4} \sinh ^{2} t-4 t \sinh \frac{t}{4} \sinh \frac{5 t}{4} \cosh t \sinh t .
\end{aligned}
$$

We clearly see that it is enough to prove $h_{2}(t)>0$ for $t>0$.

Making use of 'product to sum' and power series formulas we get

$$
\begin{aligned}
h_{2}(t)= & -t \sinh \frac{t}{2}+\frac{1}{4} \cosh \frac{t}{2}-\frac{1}{2} \cosh \frac{3 t}{2}+t^{2} \cosh \frac{3 t}{2}-t \sinh \frac{3 t}{2}+\frac{1}{4} \cosh \frac{7 t}{2} \\
& +\frac{1}{4} \cosh t+\frac{11}{4} t \sinh t-t^{2} \cosh t-\frac{1}{4} \cosh 3 t-\frac{1}{4} t \sinh 3 t \\
= & \sum_{n=1}^{\infty} \frac{s(n)}{4^{n+1}(2 n) !} t^{2 n}
\end{aligned}
$$

where

$$
s(n)=7^{2 n}-\left(\frac{2}{3} n+1\right) 6^{2 n}+\left(64 n^{2}-80 n-18\right) 3^{2 n-2}-\left(16 n^{2}-30 n-1\right) 2^{2 n}-16 n+1
$$

It is easy to verify that $s(1)=s(2)=s(3)=0, s(4)=71,680$. Next we show that $s(n)>0$ for $n \geq 5$. To this end, we rewrite $s(n)$ as

$$
s(n)=6^{2 n} s_{1}(n)+\frac{1}{9}\left(16 n^{2}-30 n-1\right) 2^{2 n} s_{2}(n)+s_{3}(n),
$$

where

$$
s_{1}(n)=\left(\frac{7}{6}\right)^{2 n}-\left(\frac{2}{3} n+1\right)
$$




$$
\begin{aligned}
& s_{2}(n)=3^{2 n}-9 \times 2^{2 n}=\left(\frac{3}{2}\right)^{2 n}-9, \\
& s_{3}(n)=\left(48 n^{2}-50 n-17\right) 3^{2 n-2}-16 n+1 .
\end{aligned}
$$

Due to $\left(16 n^{2}-30 n-1\right)=16 n(n-2)+(2 n-1)>0$ for $n \geq 2$, it suffices to prove $s_{i}(n)>0$ for $n \geq 5, i=1,2,3$. Indeed,

$$
s_{1}^{\prime}(x)=2\left(\frac{7}{6}\right)^{2 x} \ln \frac{7}{6}-\frac{2}{3} \geq 2\left(\frac{7}{6}\right)^{10} \ln \frac{7}{6}-\frac{2}{3}=0.77 \ldots>0
$$

therefore, $s_{1}(n) \geq s_{1}(5)=20,455,153 / 60,466,176>0 ; s_{2}(n)>s_{2}(3)=153 / 64>0 ; s_{3}(n)>$ $\left(48 n^{2}-50 n-17\right)-16 n+1=48(n-2)^{2}+126(n-2)+44>0$.

This completes the proof.

Proposition 4 Let $a, b>0$ with $a \neq b$. Then for $p \in(3 / 2,2)$ we have

$$
\begin{aligned}
1 & <\frac{A^{1 / 4}(a, b) L^{3 / 4}(a, b)}{A^{1 / 4}(G, A) L^{3 / 4}(G, A)}<\frac{\sqrt{L_{p}(a, b) L_{3-p}(a, b)}}{\sqrt{L_{p}(G, A) L_{3-p}(G, A)}} \\
& <\frac{L_{3 / 2}(a, b)}{L_{3 / 2}(G, A)}<\frac{\sqrt{L(a, b) A_{2 / 3}(a, b)}}{\sqrt{L(G, A) A_{2 / 3}(G, A)}}<\frac{\sqrt{L(a, b) I(a, b)}}{\sqrt{L(G, A) I(G, A)}} \\
& <\frac{S_{5 / 4,1 / 4}(a, b)}{S_{5 / 4,1 / 4}(G, A)}<\frac{H e_{3 / 4}(a, b)}{H e_{3 / 4}(G, A)}<\frac{A_{1 / 2}(a, b)}{A_{1 / 2}(G, A)}<\frac{I_{3 / 4}(a, b)}{I_{3 / 4}(G, A)},
\end{aligned}
$$

where $G=G(a, b)$ and $A=A(a, b)$.

Proof Without loss of generality, we assume that $a<b$. Then the second inequality to the last inequality in (3.3) follows easily from Proposition 3 and Lemma 5.

Next we prove the first inequality of (3.3). Let $t=\ln \sqrt{b / a}>0$. Then it equivalent to the inequality

$$
u(t)=\frac{1}{4} \ln \cosh t+\frac{3}{4} \ln \frac{\sinh t}{t}-\frac{1}{4} \ln \frac{\cosh t+1}{2}-\frac{3}{4} \ln \frac{\cosh t-1}{\ln \cosh t}>0 .
$$

Differentiating $u(t)$ gives

$$
\begin{aligned}
u^{\prime}(t) & =\frac{3 t \sinh ^{2} t-(t+3 \sinh t \cosh t+2 t \cosh t) \ln (\cosh t)}{2 t(\sinh 2 t) \ln (\cosh t)} \\
& =\frac{t+3 \sinh t \cosh t+2 t \cosh t}{2 t(\sinh 2 t) \ln (\cosh t)} \times u_{1}(t)
\end{aligned}
$$

where

$$
u_{1}(t)=\frac{3 t \sinh ^{2} t}{t+3 \sinh t \cosh t+2 t \cosh t}-\ln (\cosh t) .
$$

Differentiating $u_{1}(t)$ leads to

$$
u_{1}^{\prime}(t)=\frac{t \sinh t}{(t+2 t \cosh t+3 \cosh t \sinh t)^{2} \cosh t} \times u_{2}(t),
$$


where

$$
u_{2}(t)=\frac{13}{2} t \cosh t-3 \sinh t+t \cosh 2 t+\frac{3}{2} \sinh 2 t+\frac{3}{2} t \cosh 3 t-3 \sinh 3 t .
$$

Making use of the power series we get

$$
\begin{aligned}
u_{2}(t)= & \frac{13}{2} \sum_{n=1}^{\infty} \frac{t^{2 n-1}}{(2 n-2) !}-3 \sum_{n=1}^{\infty} \frac{t^{2 n-1}}{(2 n-1) !}+\sum_{n=1}^{\infty} \frac{2^{2 n-2} t^{2 n-1}}{(2 n-2) !} \\
& +\frac{3}{2} \sum_{n=1}^{\infty} \frac{2^{2 n-1} t^{2 n-1}}{(2 n-1) !}+\frac{3}{2} \sum_{n=1}^{\infty} \frac{3^{2 n-2} t^{2 n-1}}{(2 n-2) !}-3 \sum_{n=1}^{\infty} \frac{3^{2 n-1} t^{2 n-1}}{(2 n-1) !} \\
= & \sum_{n=1}^{\infty} \frac{v(n)}{(2 n-1) !} t^{2 n-1}
\end{aligned}
$$

where

$$
v(n)=\left(n-\frac{7}{2}\right) 3^{2 n-1}+(n+1) 2^{2 n-1}+\left(13 n-\frac{19}{2}\right) .
$$

Clearly, $v(1)=v(2)=0, v(3)=36$ and $v(n)>0$ for $n \geq 4$. Therefore, $u_{2}(t)>0, u_{1}(t)$ is strictly increasing in $(0, \infty), u_{1}(t)>u_{1}\left(0^{+}\right)=0, u^{\prime}(t)>0$, and $u(t)>u\left(0^{+}\right)=0$ for $t>0$.

Thus the proof is finished.

\section{Sharp bounds for $A G M$}

In this section, we present several sharp bounds for the arithmetic-geometric mean AGM.

Theorem 1 can be derived from Propositions 1-4 and Lemma 7.

Theorem 1 Let $a, b>0$ with $a \neq b$. Then the inequalities

$$
\begin{aligned}
\sqrt{A(a, b) G(a, b)} & <G_{3 / 4,-1 / 4}(a, b)<S_{7 / 4,-1 / 4}(a, b)<A G M(a, b) \\
& <A^{1 / 4}(a, b) L^{3 / 4}(a, b)<\sqrt{L_{p}(a, b) L_{3-p}(a, b)} \\
& <L_{3 / 2}(a, b)<\sqrt{L(a, b) A_{2 / 3}(a, b)}<\sqrt{L(a, b) I(a, b)} \\
& <S_{5 / 4,1 / 4}(a, b)<H e_{3 / 4}(a, b)<A_{1 / 2}(a, b)<I_{3 / 4}(a, b)
\end{aligned}
$$

hold for $p \in(3 / 2,2)$.

Remark 1 We clearly see that the upper bound $A^{1 / 4} L^{3 / 4}$ for $A G M$ is better than $L_{3 / 2}$. Moreover, we have

$$
A G M(a, b)<A^{1 / 4}(G, A) L^{3 / 4}(G, A)=A_{1 / 2}^{1 / 4}(a, b) L^{3 / 4}(G, A) .
$$

\section{Theorem 2 The inequality}

$$
A G M(a, b)<A^{p}(a, b) L^{1-p}(a, b)
$$

holds for all $a, b>0$ with $a \neq b$ if and only if $p \geq 1 / 4$. 
Proof Let $x>0$ and $x \rightarrow 0^{+}$. Then (2.2) and the power series

$$
\frac{1}{A^{p}(1,1-x) L^{1-p}(1,1-x)}=1+\frac{1}{2} x+\frac{4-p}{12} x^{2}+o\left(x^{2}\right)
$$

lead to

$$
\lim _{x \rightarrow 0^{+}} \frac{\frac{1}{A G M(1,1-x)}-\frac{1}{A^{p}(1,1-x) L^{1-p}(1,1-x)}}{x^{2}}=\frac{1}{12}\left(p-\frac{1}{4}\right) .
$$

Therefore, $p \geq 1 / 4$ is the necessary condition for the inequality $\operatorname{AGM}(a, b)<A^{p}(a, b) \times$ $L^{1-p}(a, b)$ to hold for all $a, b>0$ with $a \neq b$. The sufficiency follows easily from the function $p \mapsto A^{p}(a, b) L^{1-p}(a, b)$ is strictly increasing and Theorem 1 .

Let $a, b>0$ with $a \neq b, r \in \mathbb{R}$ and $\alpha \in(0,1)$. Then we define $M_{r}\left(A(a, b), L(a, b) ; \frac{1}{4}\right)$ by

$$
M_{r}\left(A, L ; \frac{1}{4}\right)=\left(\frac{1}{4} A^{r}+\frac{3}{4} L^{r}\right)^{1 / r} \quad \text { if } r \neq 0 \text { and } M_{0}\left(A, L ; \frac{1}{4}\right)=A^{1 / 4} L^{3 / 4} \text {. }
$$

Remark 2 From Theorem 1 and (1.6) we clearly see that the double inequality

$$
M_{-1}(A(a, b), L(a, b) ; 1 / 4)<A G M(a, b)<M_{0}(A(a, b), L(a, b) ; 1 / 4)
$$

holds for all $a, b>0$ with $a \neq b$.

Moreover, making use of (2.1) and (2.2) we get

$$
\begin{aligned}
& \lim _{x \rightarrow 0^{+}} \frac{A G M(1, x)-\left(\frac{1}{4}\left(\frac{x+1}{2}\right)^{r}+\frac{3}{4}\left(\frac{x-1}{\ln x}\right)^{r}\right)^{1 / r}}{1 / \ln (1 / x)}= \begin{cases}\frac{\pi}{2}-\infty, & r \geq 0, \\
\frac{\pi}{2}-\left(\frac{3}{4}\right)^{1 / r}, & r<0,\end{cases} \\
& \lim _{x \rightarrow 0^{+}} \frac{\frac{1}{A G M(1,1-x)}-\left(\frac{1}{4}\left(\frac{2-x}{2}\right)^{r}+\frac{3}{4}\left(\frac{-x}{\ln (1-x)}\right)^{r}\right)^{1 / r}}{x^{4}}=\frac{r+1 / 10}{1,536} .
\end{aligned}
$$

Therefore, $p \leq-(\ln 4-\ln 3) /(\ln \pi-\ln 2)=-0.63 \ldots$ and $q \geq-1 / 10$ are the necessary conditions such that the double inequality

$$
M_{p}\left(A(a, b), L(a, b) ; \frac{1}{4}\right)<A G M(a, b)<M_{q}\left(A(a, b), L(a, b) ; \frac{1}{4}\right)
$$

holds for all $a, b>0$ with $a \neq b$.

Conjecture 1 The double inequality

$$
M_{p}\left(A(a, b), L(a, b) ; \frac{1}{4}\right)<A G M(a, b)<M_{q}\left(A(a, b), L(a, b) ; \frac{1}{4}\right)
$$

holds for all $a, b>0$ with $a \neq b$ if and only if $p \leq-(\ln 4-\ln 3) /(\ln \pi-\ln 2)$ and $q \geq-1 / 10$.

Theorem 3 Let $p, q>3 / 4$. Then the double inequality

$$
S_{p, 3 / 2-p}(a, b)<A G M(a, b)<S_{q, 3 / 2-q}(a, b)
$$

holds for all $a, b>0$ with $a \neq b$ if and only if $p \geq 7 / 4$ and $3 / 4<q \leq 3 / 2$. 
Proof The sufficiency follows from the function $p \mapsto S_{p, 3 / 2-p}(a, b)$ is strictly decreasing in $(3 / 4, \infty)$ by Lemma 2 and Theorem 1 .

Next we prove the necessity. It follows from (2.1) and (2.2) together with the power series

$$
\frac{1}{S_{p, 3 / 2-p}(1,1-x)}=1+\frac{1}{2} x+\frac{5}{16} x^{2}+\frac{7}{32} x^{3}+\frac{2 p^{2}-3 p+316}{1,920} x^{4}+o\left(x^{4}\right)
$$

that

$$
\begin{aligned}
& \lim _{x \rightarrow 0^{+}} \frac{\frac{1}{A G M(1,1-x)}-\frac{1}{S_{p, 3 / 2-p}(1,1-x)}}{x^{4}}=\frac{169}{1,024}-\frac{2 p^{2}-3 p+316}{1,920}, \\
& \lim _{x \rightarrow 0^{+}} \frac{A G M(1, x)-S_{q, 3 / 2-q}(1, x)}{1 /(\ln (1 / x))}= \begin{cases}\frac{\pi}{2}-\infty, & 3 / 4<q \leq 3 / 2, \\
\frac{\pi}{2}, & q>3 / 2 .\end{cases}
\end{aligned}
$$

Therefore, $p \geq 7 / 4$ and $3 / 4<q \leq 3 / 2$ are the necessary conditions the double inequality (4.4) to hold for all $a, b>0$ with $a \neq b$.

Theorem 4 Let $p \geq 1 / 2$. Then the inequality

$$
\operatorname{AGM}(a, b)<\sqrt{S_{p, 1}(a, b) S_{1-p, 1}(a, b)}
$$

holds for all $a, b>0$ with $a \neq b$ if and only if $1 / 2 \leq p \leq 1$.

Proof The sufficiency follows from the function $p \mapsto \sqrt{S_{p, 1}(a, b) S_{1-p, 1}(a, b)}$ is strictly decreasing in $(1 / 2, \infty)$ by Corollary 2 (ii) and the inequality

$$
\operatorname{AGM}(a, b)<\sqrt{L(a, b) I(a, b)}=\sqrt{S_{1,1}(a, b) S_{0,1}(a, b)}
$$

in Theorem 1, and the necessity can be derived from the inequality

$$
\lim _{x \rightarrow 0^{+}} \frac{A G M(1, x)-\sqrt{S_{p, 1}(1,1-x) S_{1-p, 1}(1,1-x)}}{1 /(\ln (1 / x))}= \begin{cases}\frac{\pi}{2}-\infty, & 1 / 2 \leq p \leq 1, \leq 0 . \\ \frac{\pi}{2}, & p>1\end{cases}
$$

Making use of the similar methods, we can prove Theorems 5-7, we omit the proofs here.

Theorem 5 Let and $p, q>1 / 4$. Then the double inequality

$$
G_{p, 1 / 2-p}(a, b)<A G M(a, b)<G_{q, 1 / 2-q}(a, b)
$$

holds for all $a, b>0$ with $a \neq b$ if and only if $p \geq 3 / 4$ and $1 / 4<q \leq 1 / 2$.

Theorem 6 The double inequality

$$
S_{p+1, p}(a, b)<A G M(a, b)<S_{q+1, q}(a, b)
$$

holds for all $a, b>0$ with $a \neq b$ if and only if $p \leq 0$ and $q \geq 1 / 4$. 
Theorem 7 The double inequality

\author{
$\operatorname{He}_{p}(a, b)<A G M(a, b)<H e_{q}(a, b)$ \\ holds for all $a, b>0$ with $a \neq b$ if and only if $p \leq 0$ and $q \geq 3 / 4$.
}

Competing interests

The authors declare that they have no competing interests.

\title{
Authors' contributions
}

All authors contributed equally to the writing of this paper. All authors read and approved the final manuscript.

\section{Acknowledgements}

This research was supported by the Natural Science Foundation of China under Grants 11371125, 11171307, and 61374086, and the Natural Science Foundation of Zhejiang Province under Grant LY13A010004.

Received: 17 December 2013 Accepted: 29 April 2014 Published: 14 May 2014

\section{References}

1. Borwein, JM, Borwein, PB: Pi and the AGM. Wiley, New York (1987)

2. Stolarsky, KB: Generalizations of the logarithmic mean. Math. Mag. 48, 87-92 (1975)

3. Gini, C: Di una formula comprensiva delle medie. Metron 13(2), 3-22 (1938)

4. Carlson, BC, Vuorinen, M: Inequality of the AGM and the logarithmic mean. SIAM Rev. 33(4), 653-654 (1991)

5. Sándor, J: On certain inequalities for means. J. Math. Anal. Appl. 189(2), 602-606 (1995)

6. Qi, F, Sofo, A: An alternative and united proof of a double inequality for bounding the arithmetic-geometric mean. Sci. Bull. "Politeh." Univ. Buchar., Ser. A, Appl. Math. Phys. 71(3), 69-76 (2009)

7. Neuman, E, Sándor, J: On certain means of two arguments and their extensions. Int. J. Math. Math. Sci. 16, 981-993 (2003)

8. Yang, Z-H: A new proof of inequalities for Gauss compound mean. Int. J. Math. Anal. 4(21-24), 1013-1018 (2010)

9. Vamanamurthy, MK, Vuorinen, M: Inequalities for means. J. Math. Anal. Appl. 183(1), 155-166 (1994)

10. Borwein, JM, Borwein, PB: Inequalities for compound mean iterations with logarithmic asymptotes. J. Math. Anal. Appl. 177(2), 572-582 (1993)

11. Alzer, H, Qiu, S-L: Monotonicity theorems and inequalities for the complete elliptic integrals. J. Comput. Appl. Math. 172(2), 289-312 (2004)

12. Qiu, S-L, Shen, J-M: On two problems concerning means. J. Hangzhou Inst. Electron. Eng. 17(3), 1-7 (1997) (in Chinese)

13. Sándor, J: On certain inequalities for means II. J. Math. Anal. Appl. 199(2), 629-635 (1996)

14. Sándor, J: On certain inequalities for means III. Arch. Math. 76(1), 34-40 (2001)

15. Toader, G: Some mean values related to the arithmetic-geometric mean. J. Math. Anal. Appl. 218(2), 358-368 (1998)

16. Chung, S-Y: Functional means and harmonic functional means. Bull. Aust. Math. Soc. 57(2), 207-220 (1998)

17. Bracken, P: An arithmetic-geometric mean inequality. Expo. Math. 19(3), 273-279 (2001)

18. Liu, Z: Compounding of Stolarsky means. Soochow J. Math. 30(2), 149-163 (2004)

19. Liu, Z: Remarks on arithmetic-geometric mean and geometric-harmonic mean. J. Anshan Univ. Sci. Technol. 30(3), 230-235 (2007) (in Chinese)

20. Neuman, E: Inequalities for weighted sums of powers and their applications. Math. Inequal. Appl. 15(4), 995-1005 (2012)

21. Yang, Z-H: The log-convexity of another class of one-parameter means and its applications. Bull. Korean Math. Soc. 49(1), 33-47 (2012)

22. Losonczi, L: Ratio of Stolarsky means: monotonicity and comparison. Publ. Math. (Debr.) 75(1-2), 221-238 (2009)

23. Yang, Z-H: Some monotonicity results for the ratio of two-parameter symmetric homogeneous functions. Int. J. Math Math. Sci. 2009, Article ID 591382 (2009)

24. Yang, Z-H: Log-convexity of ratio of the two-parameter symmetric homogeneous functions and an application. J. Inequal. Spec. Funct. 1(1), 16-29 (2010)

25. Yang, Z-H: The monotonicity results for the ratio of certain mixed means and their applications. Int. J. Math. Math. Sci. 2012, Article ID 540710 (2012)

26. Peetre, J: Generalizing the arithmetic geometric mean - a hapless computer experiment. Int. J. Math. Math. Sci. 12(2), 235-245 (1989)

10.1186/1029-242X-2014-192

Cite this article as: Yang et al.: Sharp bounds for the arithmetic-geometric mean. Journal of Inequalities and Applications 2014, 2014:192 